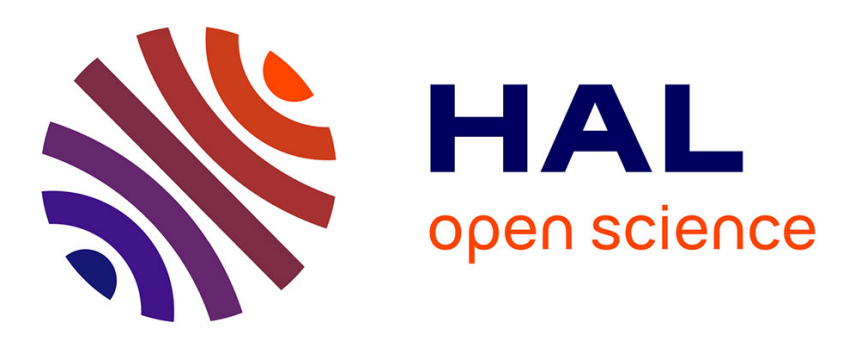

\title{
A comprehensive study of bubble inflation in vacuum-assisted thermoforming based on whole-field strain measurements
}

\author{
A. Ayadi, M.-F. Lacrampe, P. Krawczak
}

\section{- To cite this version:}

A. Ayadi, M.-F. Lacrampe, P. Krawczak. A comprehensive study of bubble inflation in vacuum-assisted thermoforming based on whole-field strain measurements. 21st International ESAFORM Conference on Material Forming (ESAFORM 2018), Apr 2018, Palermo, Italy. pp.120004, 10.1063/1.5034972 . hal-01801996

\section{HAL Id: hal-01801996 \\ https://hal.science/hal-01801996}

Submitted on 28 May 2018

HAL is a multi-disciplinary open access archive for the deposit and dissemination of scientific research documents, whether they are published or not. The documents may come from teaching and research institutions in France or abroad, or from public or private research centers.
L'archive ouverte pluridisciplinaire HAL, est destinée au dépôt et à la diffusion de documents scientifiques de niveau recherche, publiés ou non, émanant des établissements d'enseignement et de recherche français ou étrangers, des laboratoires publics ou privés. 


\title{
A comprehensive study of bubble inflation in vacuum- assisted thermoforming based on whole-field strain measurements
}

\author{
A. Ayadi 1, 2, a) M-F. Lacrampe ${ }^{1,2, b)}$ and P. Krawczak ${ }^{1,2}$ \\ ${ }^{1}$ IMT Lille Douai, Institut Mines-Télécom, Polymers and Composites Technology \& Mechanical Engineering \\ Department, 941 rue Charles Bourseul, 59508 Douai, France. \\ ${ }^{2}$ Université de Lille, 59000 Lille, France. \\ ${ }^{a}$ Presenting author: abderrahmane.ayadi@imt-lille-douai.fr \\ ${ }^{b)}$ Corresponding author: marie-france.lacrampe @imt-lille-douai.fr
}

\begin{abstract}
This paper focuses on the potential use of stereo-DIC in thermoforming conditions to monitor large deformations of softened thermoplastic sheets posteriori to the sagging phenomenon. The study concerns HIPS sheets which are softened by the radiative heat-transfer mode then stretched by inflation of compressed-air for $1.5 \mathrm{~s}$ to form a large and quasi-spherical dome of $250 \mathrm{~mm}$ in diameter. While the bubble-inflation operation leads to large deformations of the softened sheet, it shows transitional geometrical instabilities due to the initial surface sagging. When the temperature-induced surface deformations are inaccessible by the stereoscopic system during the heating operation, the geometrical instabilities limit the identification of the reference of displacements which affects the accuracy of results based on image-correlation computations. To compare between the principal strains assessed from bubble-inflation tests conducted at different thermal conditions, a method for filtering these instabilities is developed in this study.
\end{abstract}

\section{INTRODUCTION}

Experimental characterization of the mechanical behavior of thermoplastics in the range of thermoforming temperatures is classically performed based on unidirectional and multi-axial tests performed at controlled deformation speeds at isothermal conditions. However, the optimization of thermoforming simulation packages requires the development of original mechanical tests which are more representative of the material behavior under processing conditions. One potential solution is the use of open-mold thermoforming equipment and full-field strain computation techniques to characterize the thermomechanical behavior of thermoplastics. In the field of experimental mechanics, the whole-field optical techniques have an advantage of being fast and contact-less [1,2]. In particular, stereo-DIC is more and more applied in thermoforming to compute whole-field strains [3], investigate thermoformability limits [4] and compute full-field thickness maps [5]. During a vacuum-assisted thermoforming cycle, thermoplastic sheets are thermally softened then pre-stretched by forming a quasi-spherical bubble to homogenize their thicknesses. During the bubble-inflation operation, the interaction between the non-conditioned blown air and the softened sheet does not only exhibit large deformations but also causes geometrical non-linearity due to the stochastic thermal deformations taking place during the heating operation. In fact, the sheets are mainly softened based on the radiative heat-transfer mode which inevitably induces warpage of the surface due to relaxation of the residual stresses and descent of the softened surface under the effect of its own weight. The latter phenomenon is known by sag in thermoforming. When the access to the sheet's surface during heating by the stereoscopic system is not possible, an appropriate protocol is required to avoid erroneous interpretations of the stereo-DIC results relative to the large deformations taking place during the bubble formation. This study provides an overview of the potential use of stereo-DIC measurements in thermoforming conditions, particularly during a pre-stretching operation of $1.5 \mathrm{~s}$ 
by bubble inflation of High-Impact Polystyrene sheets (HIPS). This case focuses on the applicability of stereo-DIC measurements when the monitoring of large deformations can only be possible after thermal sagging of the surface. First, the constraints imposed by the equipment that affect the accuracy of stereo-DIC computations are presented. Second, an objective stereo-DIC post-processing method is presented to evaluate the principle strains from stereoDIC measurements during bubble-inflation tests which are performed after two heating temperatures. With access to the temperature distributions of the HIPS sheet after heating, full-field principle strains will be used to understand the effect of sagging on the resulting wall-thickness distributions.

\section{EXPERIMENTAL PROTOCOL}

In this study, bubble-inflation tests are performed by using a thermoforming machine of the type Illig UA-100Ed equipped with a clamp-frame which is designed to form a semi-spherical bubble of $250 \mathrm{~mm}$ in diameter. The used HIPS sheets are commercialized under the trade name Athpol P91® and have an average thickness of $1.5 \mathrm{~mm}$. Before each thermoforming cycle, a sheet of $600 \times 330 \mathrm{~mm}^{2}$ is cut to meet the dimensions of the forming plateau and a black and white speckle is applied on its surface as required for stereo-DIC experiments. The thermoforming cycle starts automatically by clamping the sheet to seal a cavity located below the forming plateau of the machine. Then, a sliding radiative heater entirely covers the forming plateau to heat the fixed sheet at a defined regulation temperature and for a fixed duration of $70 \mathrm{~s}$. The heater comprises a battery of infra-red (IR) ceramic elements of the type Acim Jouanin ${ }^{\circledR}$ which are located at a distance of $150 \mathrm{~mm}$ from the surface of the clamped HIPS sheet. After the complete retraction of the heater (within less than $2 \mathrm{~s}$ ), compressed-air is inflated inside the sealed cavity covered by the softened sheet for $1.5 \mathrm{~s}$ to force the softened thermoplastic sheet through the circular opening in the clamping-frame. The realized experimental plan comprised a total of 10 bubble-inflation tests which are carried out at the regulation temperatures of $310^{\circ} \mathrm{C}$ and $330{ }^{\circ} \mathrm{C}$.

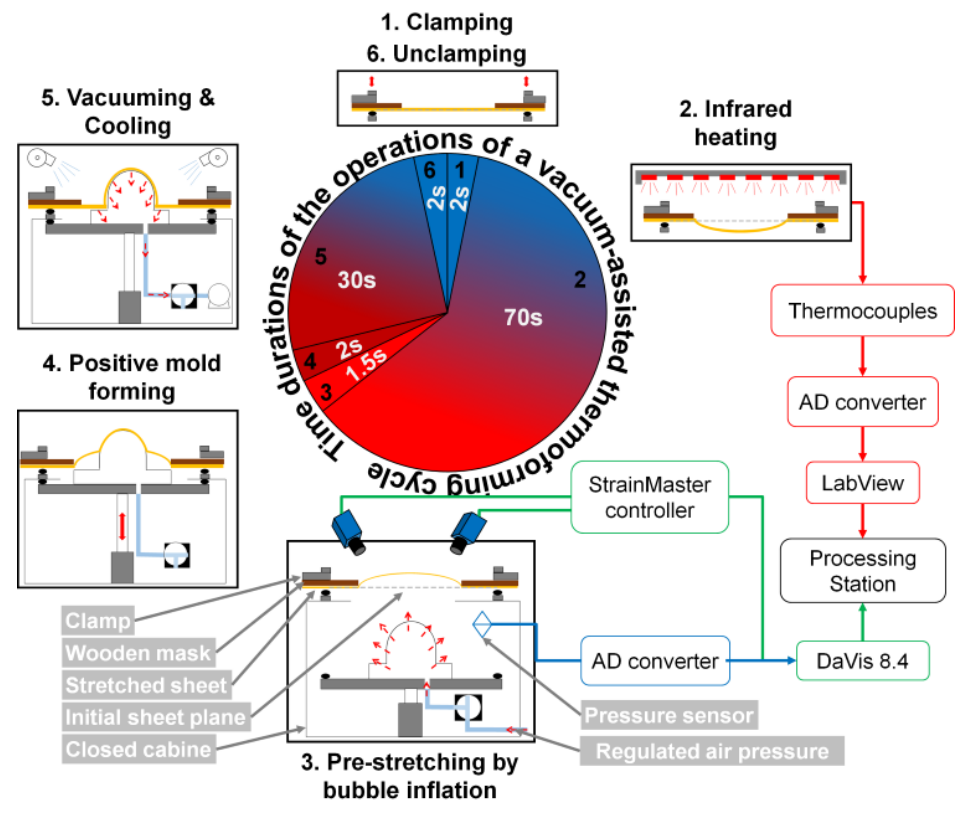

FIGURE 1. Overview of the used experimental configuration combining stereo-DIC and thermoforming.

Because the used machine does not provide continuous time monitoring of the process parameters such as the material's temperature and air pressure, external acquisition devices are installed. K-type thermocouples are used to measure temperatures during the heating step on the surface of thermoplastic sheets. A pressure sensor of the type JUMO AP-30R is used to measure the relative pressure inside the bubble during the air-inflation operation. The evolution of the surface speckle is observed from different angles by using two identical CMOS cameras (Imager MX 4M) which are calibrated to make stereoscopic measurements within a common coordinates system. During the bubble-inflation operation, the pressure signal is synchronized with a high-speed-controller of a stereo-DIC system commercialized by LaVision. Pairs of images are recorded at a full-resolution of $2048 \times 2048$ pixels and a frequency of $180 \mathrm{fps}$. An overview of the experimental setup is illustrated in Fig.1. 


\section{CONSTRAINTS IMPOSED BY THE THERMOFORMING EQUIPMENT}

\section{Existence of Temperature Gradients after the Material Heating}

During the radiative heating operation, a circular domain of the HIPS sheet of $250 \mathrm{~mm}$ in diameter is exposed to the IR ceramic elements. Because the machine does not provide direct measurements of the thermoplastic temperature at the end of the heating operations, an experimental procedure based on the Kriging interpolation method is used to evaluate the temperature distributions within a surface domain covering $100 \times 120 \mathrm{~mm}^{2}$. As illustrated in Fig.2, the obtained temperature maps indicate the existence of significant in-plane thermal gradients. Such observation reflects a non-uniform heating despite the automatic regulation of the heater's temperature. These temperature distributions confirm the existence of local changes of the surface flatness and notably sagging which are both known to affect the thermal performance of the IR heaters.

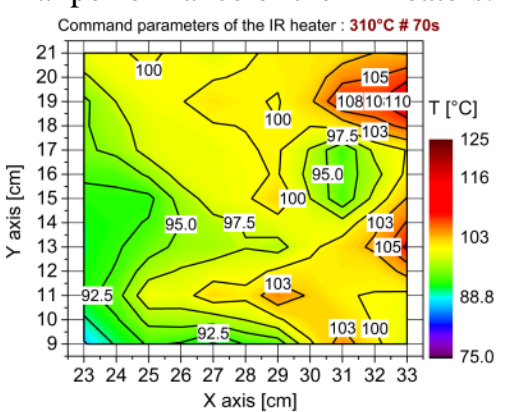

(a)

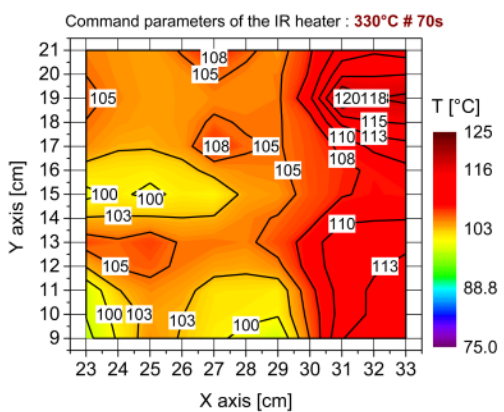

(b)

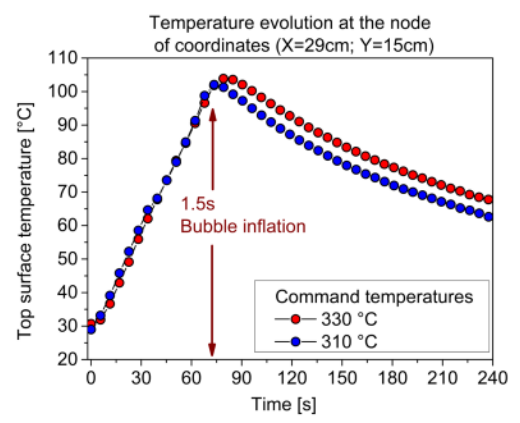

(c)

FIGURE 2. a-b) Temperature maps at the surface of HIPS sheets based on Kriging interpolation method. c) Temperature profiles measured during IR heating at the position of coordinates $(X=29 \mathrm{~cm} ; Y=15 \mathrm{~cm})$.

\section{Large Deformations and Speckle Uniformity Considerations}

The used thermoforming cycle gives the possibility to deform a softened thermoplastic sheet at two successive stages. First, the pressure build-up during the inflation of compressed-air inside the sealed cabinet forces the softened HIPS sheet through the relatively large circular opening of the clamp-frame. The height of the thermoplastic sheet at the end of this step is limited to few centimeters and it can be calibrated with variation of the heater's regulation temperature (domain between B and C in Fig.3.a). To avoid exceeding the depth-of-field of the used cameras, the control temperatures were limited to $330{ }^{\circ} \mathrm{C}$ and $310^{\circ} \mathrm{C}$ resulting in bubble heights lower than $1 / 4$ of the diameter. This height threshold is defined based on trial tests and visual inspections of deformed surfaces to check the uniformity of the speckle and absence of paint cracking at the end of the bubble formation. In the second deformation stage, a forming table comprising a positive punch of $200 \mathrm{~mm}$ in diameter moves upwards starting from the bottom of the forming cabinet to deform the sheet for a maximum height of $250 \mathrm{~mm}$ (domain between $\mathrm{C}$ and $\mathrm{E}$ in Fig.3.a). A latency duration of almost two seconds marks the time required for the punch to reach the surface of the inflated bubble (domain between C and D in Fig.3.a).

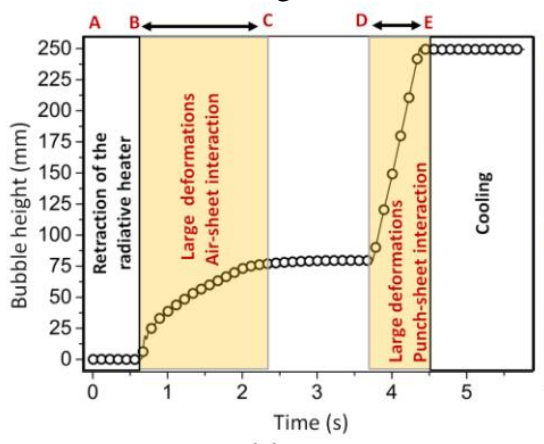

(a)

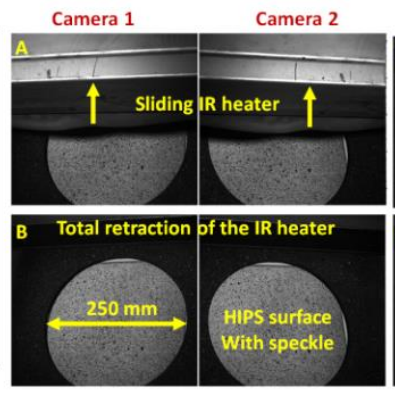

(b)

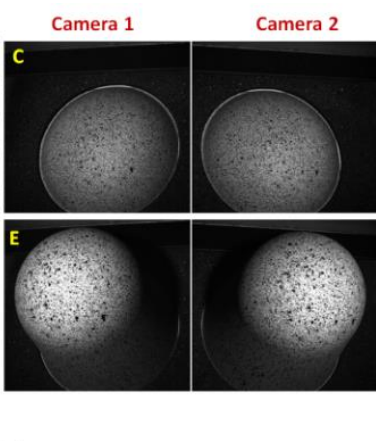

FIGURE 3. a) Bubble-height variations during large deformations obtained by the used thermoforming equipment. b) Stereo images of the observed field-of-view at the representative moments corresponding to the moments $\mathrm{A}, \mathrm{B}, \mathrm{C}$ and $\mathrm{E}$. 
With consideration of the large extent of the analyzed surface and the calibration constraints of the used stereoscopic system (such as the variations of luminosity as illustrated in Fig.3.b), only deformations taking place by the interaction between compressed-air and the softened thermoplastic sheet are considered in this study.

\section{Limited Access to the Speckle During Sagging}

During the heating operation, the sliding IR heater totally covers the thermoplastic sheet (Fig.3.b). Consequently, the potential deformations of the speckle due the collapse of the softened sheet under the effect of its weight and due to the relaxation of residual stresses cannot be observed by the used stereoscopic system. Thus, the initially flat surface of the HIPS sheet should no longer be considered as a reference for stereo-DIC computations. Moreover, the temperature-induced deformations are dependent of the thermal history of the used thermoplastic sheet (extrusion direction, calendaring ...) and the homogeneity of the temperature distributions on its surface. Thus, different sag levels are obtainable even posteriori to similar heating conditions. Consequently, the identification of a reference state of the speckle (which is required for the computation of displacements and strains) requires an objective method to avoid errors and misinterpretations caused by the random and uncontrolled aspects of the thermally activated deformations.

\section{POST-PROCESSING METHOD FOR STEREO-DIC COMPUTATIONS}

To filter the out-of-plane undulations that affect the accuracy of the stereo-DIC computations, the developed approach consists on using a two-step computation. The first step is based on the assumption that the speckle of the sagged surface represents the reference for the displacement vectors. To identify the moment of attenuation of the undulations, the exploitation of the computed displacements is limited to qualitative analyses of their temporal evolution. The second step consists on correcting the reference of the displacement and deformation fields after filtering the surface instabilities. The aim of this study is to propose an objective and repetitive method to filter surface undulations regardless of the temperature of the test. The commercial software DaVis 8.4 is used to compute the displacements using the sum of the differential algorithms. The stereo-DIC computations are limited to the polar domain of the bubbles defined by a virtual gauge of $50 \mathrm{~mm}$ radius concentric to the circular opening of the used clamping frame. The size of the subset and the step size were respectively $81 * 81$ pixels and $21 * 21$ pixels.

\section{Qualitative Analysis of the Geometrical Instability}

In the first step, the reference state of the speckle is attributed to the onset of bubble inflation based on the measured pressure signals. The assessed average out-of-plane displacements of two representative tests performed at $310^{\circ} \mathrm{C}$ and $330{ }^{\circ} \mathrm{C}$ and their corresponding measured pressures are presented in Fig.4.a. The temporal evolution of the out-ofplane components of displacements indicates the existence of two regimes. An initial transient regime of nearly 300 ms which presents local maxima and minima (between $100 \mathrm{~ms}$ and $250 \mathrm{~ms}$ ) corresponding to the transition from the sagged to the strained geometries of the softened sheet. The second regime is more uniform and represents the effective extension of the thermoplastic sheet. The repetitiveness of formation of the irregular out-of-plane displacement transitions is verified by comparing the out-of-plane displacements from five repetitive bubble-inflation tests. Similar aspects are observed in all the carried-out bubble-inflation tests. However, the amplitude and the surface transition are more significant at the regulation temperature of $330{ }^{\circ} \mathrm{C}$. As an illustrative example, the out-of-plane components of displacement vectors relative to the inflation tests that were conducted after a heating temperature of $330\left({ }^{\circ} \mathrm{C}\right)$ are provided in Fig.4.b.

\section{Identification of the Effective Reference for Stereo-DIC computations}

With consideration of the used image recording frequency of $180 \mathrm{fps}$, the out-of-plane displacements at the pole of the bubble are assumed as differentiable functions of time. The time derivatives of displacements are expressed in discretized form as expressed in Eq.1:

$$
\frac{\partial w}{\partial t} \equiv \lim _{t_{i+1} \rightarrow t_{i}}\left(\frac{w_{i+1}-w_{i}}{t_{i+1}-t_{i}}\right) \cong 180 \cdot\left(w_{i+1}-w_{i}\right)
$$


Where: $w$ and $t$ indicate respectively the out-of-plane components of the displacement vectors and time increment corresponding to the two successive stereoscopic-image pairs of the incremental orders $i$ and $i+1$.

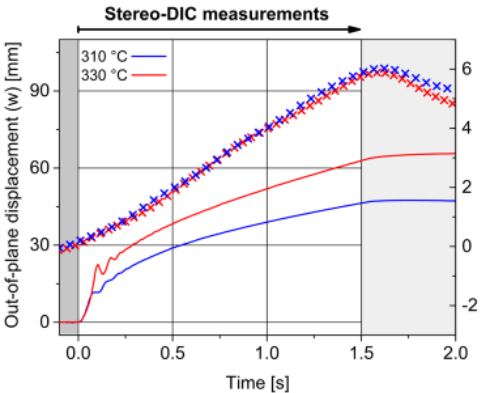

(a)

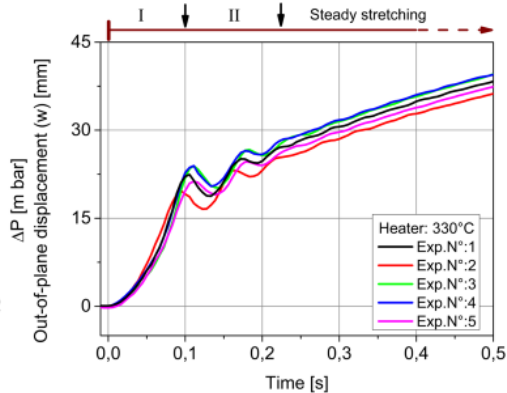

(b)

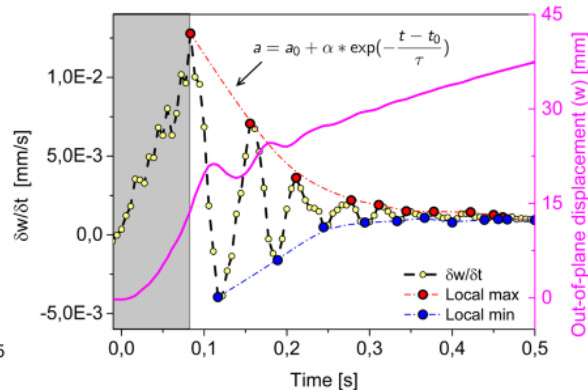

(c)

FIGURE 4. a) Pressure and average out-of-plane displacement evolution during bubble inflation. b) Reproducibility of the displacement instabilities of the tests conducted at $330^{\circ} \mathrm{C}$. c) Suggested undulation filtering method.

The inflation test conducted at $330^{\circ} \mathrm{C}$ and referenced by Exp. $n^{\circ} 5$ is considered as a representative case. The corresponding out-of-plane and computed derivatives as function of time are reported in Fig.4.c. The transitional domain of displacements (during about $300 \mathrm{~ms}$ ) corresponds to an alternation between local maxima and minima of the derivative. Such variations can be explained by the opposing effects of gravity and the fast pressure increase inside the cavity of the forming cabinet. A decayed exponential analytical form as expressed in (Eq.2) is selected to fit the time decrease of local maxima of the derivatives:

$$
\mathrm{a}=\mathrm{a}_{0}+\alpha \cdot \exp \left[-\frac{\left(\mathrm{t}-\mathrm{t}_{0}\right)}{\tau}\right]
$$

Where: $\mathrm{t}_{0}, \mathrm{a}_{0}, \alpha$ and $\tau$ respectively referred to a time offset, an amplitude offset, a pre-exponential coefficient and characteristic time duration. The results of the fitting operations are presented in Table.1.

TABLE 1. Identified parameters using the expression in Eq. 2

\begin{tabular}{ccccccccc}
\hline Test & \multicolumn{9}{c}{$\mathbf{3 1 0}^{\circ} \mathbf{C}$} \\
Ref. & $\mathbf{a}_{\mathbf{0}}(\mathbf{m m} / \mathbf{s})$ & $\boldsymbol{\alpha}(\mathbf{m m} / \mathbf{s})$ & $\mathbf{t}_{\mathbf{0}}(\mathbf{s})$ & $\boldsymbol{\tau}(\mathbf{s})$ & $\mathbf{a 0}_{\mathbf{0}}(\mathbf{m m} / \mathbf{s})$ & $\boldsymbol{\alpha}(\mathbf{m m} / \mathbf{s})$ & $\mathbf{t}_{\mathbf{0}}(\mathbf{s})$ & $\boldsymbol{\tau}(\mathbf{s})$ \\
\hline $\mathbf{1}$ & 13.636 & 127.912 & 0.738 & 0.215 & 27.586 & 370.775 & 0.750 & 0.050 \\
$\mathbf{2}$ & 12.031 & 125.812 & 0.789 & 0.217 & 24.948 & 305.372 & 0.714 & 0.074 \\
$\mathbf{3}$ & 14.025 & 137.466 & 0.560 & 0.201 & 28.473 & 388.115 & 0.702 & 0.050 \\
$\mathbf{4}$ & 15.121 & 126.166 & 0.561 & 0.214 & 27.772 & 347.009 & 0.684 & 0.051 \\
$\mathbf{5}$ & 14.123 & 132.499 & 0.550 & 0.213 & 27.224 & 334.630 & 0.827 & 0.051 \\
Average & 13.787 & 129.971 & 0.640 & 0.212 & 27.201 & 349.180 & 0.735 & 0.055 \\
Std & 1.006 & 4.440 & 0.103 & 0.006 & 1.197 & 28.698 & 0.051 & 0.009 \\
\hline
\end{tabular}

The characteristic time-durations $(\tau)$ relative to bubble-inflation tests conducted at identical heating temperatures present similar order of magnitudes. The advantage of using of a decayed exponential form is related to the significance of the characteristic time duration which corresponds to the duration required to the attenuation of $63 \%$ of the maximal velocity amplitude at the origin of times. Consequently this parameter can be considered as an objective indicator to select the reference of the stereo-DIC computations, regardless of the initial sagging level.

\section{Conversion of Principle Strains to Thickness Maps}

Under the assumption of material incompressibility, which is reported in different studies in the case of HIPS, the maximum and minimum deformation fields on the surface can be used to compute full-fields to thicknesses. Such information is helpful especially to adjust the heating levels and to evaluate the influence of any temperature heterogeneous distribution on the resulting quality of the pre-stretching phase. According to the formalism detailed in [5] the thickness descriptions are not influenced with the adopted definition of the strain fields. We note that the stereoDIC technique was successfully used to image the whole circular region of the plate that was exposed to heater. However, to be coherent with the evaluated temperature maps based on the Kriging interpolation method, only thinning distributions within the mapped zones are presented in Fig.5. Comparison between Fig.2 and Fig.5 indicates 
similarities between the distributions of temperatures and thinning levels. The highest thinning levels are measured at the zones of highest surface temperatures.

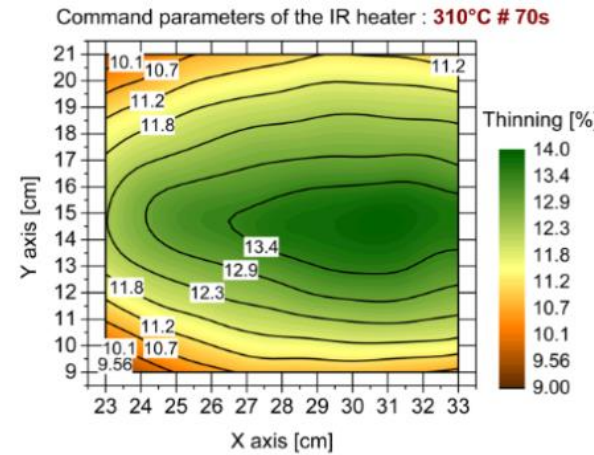

(a)

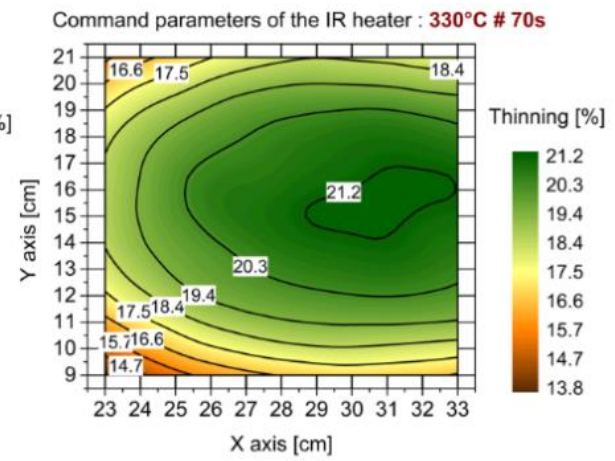

(b)

FIGURE 5. Thinning distributions at the end of a $1.5 \mathrm{~s}$ bubble inflation operation. a) Command temperature of the heater at $310^{\circ} \mathrm{C}$. b) Command temperature of the heater at $330^{\circ} \mathrm{C}$.

\section{CONCLUSIONS}

By using the Kriging interpolation method during the radiative heating of thin HIPS sheets, the existence of surface sagging was detected from the presence of temperature gradients. To quantify the actual stretching of the sagged surface that takes place during bubble-inflation by stereo-DIC, an objective identification of the reference of deformations was required. Stereoscopic measurements performed at a frequency of 180 fps during bubble-inflation posteriori to two different heating conditions showed that the geometrical instabilities corresponded to out-of-plane damping undulations of the sheet. The time derivatives of the out-of-plane displacements confirmed that the transition moment from the sagged to the initially strained geometries of the sheet corresponded to the attenuation of $63 \%$ of their maximal velocity amplitude, regardless of the initial sagging level. The developed method allowed comparison between the principal strains that were assessed from different thermal conditions and correlation of the surface thinning with the initial temperature distributions of the softened HIPS sheets. This work constitutes a first step towards using open-mold thermoforming equipment and stereo-DIC based strain measurements to identify the thermomechanical parameters of thermoplastic sheets.

\section{ACKNOWLEDGEMENTS}

The authors acknowledge the European Regional Development Fund FEDER, the French state and the Hauts-deFrance Region council for co-funding the ELSAT 2020 by CISIT project (POPCOM action).

\section{REFERENCES}

1. Y. Li, J. A. Nemes and A. Derdouri, Opt. Lasers Eng. 33, 261-76 (2000).

2. D. Xu and K. M. Liechti, Exp. Mech. 50, 217-25 (2010).

3. B. Van Mieghem, F. Desplentere, A. Van Bael and J. Ivens, Express Polym. Letters 9, 119-28 (2015).

4. N. A. Zanjani, A. Sexton and S. Kalyanasundaram, Compos. Part A 68, 251-63 (2015).

5. B. Van Mieghem, J. Ivens, and A. Van Bael, Exp. Tech. 40,1409-20 (2016). 